\title{
Neuropathie motrice périphérique et maladie de Pompe : association fortuite?
}

\author{
Céline Tard, François Cassim, Luc Defebvre, Patrick Vermersch, Roseline Froissart, Magali \\ Pettazzoni, Thierry Perez, Emmanuelle Jaillette, Sylvie Nguyen
}

Le cas suivant a été présenté le 20 mars 2019 à Paris lors de la Journée Annuelle de la Maladie de Pompe. Il s'agit d'un patient admis en réanimation pour une détresse respiratoire aiguë liée à une atteinte sévère du muscle diaphragmatique. L'EMG réalisé lors de cette première hospitalisation était compatible avec une neuropathie motrice démyélinisante auto-immune. Quelques années plus tard, devant la persistance de CPK subnormales et d'un tableau respiratoire toujours sévère, un dosage de l'activité maltase acide a été proposé. Le diagnostic de maladie de Pompe est alors posé.

\author{
Céline Tard \\ François Cassim \\ Luc Defebvre \\ Patrick Vermersch \\ Sylvie Nguyen \\ CHU de Lille, Centre \\ de référence des maladies \\ neuromusculaires Nord \\ Est Île-de-France, Clinique \\ de neurologie, Lille, \\ France \\ Roseline Froissart \\ Magali Pettazzoni \\ Hospices civils de Lyon, \\ Laboratoire des Maladies \\ Héréditaires du \\ Métabolisme, Bron, \\ France \\ Thierry Perez \\ CHU de Lille, Centre \\ de référence des maladies \\ neuromusculaires Nord \\ Est Île-de-France, \\ Explorations \\ fonctionnelles \\ respiratoires, Lille, France \\ Emmanuelle Jaillette \\ CHU de Lille, Centre \\ de référence des maladies \\ neuromusculaires Nord \\ Est Île-de-France, Pôle de \\ réanimation, Lille, France \\ Contact \\ celine.tard@chru-lille.fr
}

\section{Observation}

En 2005, un patient âgé de 54 ans aux antécédents de déficience intellectuelle légère (attribuée à une méningite survenue vers l'âge de 3 ans) et tabagique à 30 paquets-années sevré était admis en réanimation pour un tableau de coma non traumatique révélateur d'une encéphalopathie hypercapnique avec détresse respiratoire aigüe : désaturation à $70 \%$ en air ambiant et acidose respiratoire décompensée (pH à 7,18; $\mathrm{PCO}_{2}$ à $108 \mathrm{mmHg} ; \mathrm{PO}_{2}$ à $34 \mathrm{mmHg}$, $\mathrm{SAO}_{2}$ à $54 \%$ ) nécessitant une ventilation invasive. Aucune cause cardio-pulmonaire n'était retrouvée. Devant l'échec d'extubation, une trachéotomie chirurgicale fut réalisée le mois suivant. La spirométrie réalisée sur canule de trachéotomie retrouvait un syndrome restrictif sévère (avec une capacité vitale à $22 \%$ de la théorique) et une difficulté d'adaptation à l'effort sous forme d'une diminution du volume minute maximal.

L'IRM cérébrale était normale ainsi que la ponction lombaire (pas d'hyperprotéinorrachie notamment). L'EMG montrait une réduction bilatérale de l'amplitude des réponses motrices diaphragmatiques sans allongement des latences.

On notait sur le plan moteur une discrète faiblesse de la pince pouce-index gauche. La sensibilité était normale. Il existait une aréflexie ostéotendineuse aux quatre membres. L'examen des nerfs crâniens était normal. Il n'y avait pas de troubles sphinctériens. En reprenant l'anamnèse, il avait déjà depuis quelques mois une fatigabilité à la marche, des crampes quadricipitales et une dyspnée.

L'EMG réalisé un mois après l'épisode aigu était compatible avec une neuropathie auto-immune, de type neuropathie motrice multifocale à blocs de conduction (Tableau 1). Les PES étaient normaux, les PEM retrouvaient une souffrance majeure de la voie motrice à destinée des deux membres inférieurs avec temps de conduction centrale incalculable. Un bilan de neuropathie auto-immune secondaire avait été fait, négatif. Les anticorps anti-onconeuronaux et anti-gangliosides étaient négatifs.

L'état du patient s'est ensuite stabilisé : ventilation spontanée sur trachéotomie la journée, ventilation invasive la nuit ou lors du décubitus avec orthopnée sévère. Le déficit moteur était global, symétrique, coté entre 3 et $4 / 5$ aux quatre membres avec une prédominance au niveau des extenseurs du genou et des fléchisseurs de la hanche. Les réflexes tendineux restaient abolis à l'exception du réflexe tricipital droit. Il n'y avait pas d'atteinte sensitive ni des nerfs crâniens. La capacité vitale restait très altérée à $26 \%$ de la théorique.

Le patient avait repris la marche avec dandinement et steppage après la première cure d'immunoglobulines. Les cures ont été poursuivies toutes les 8 semaines avec une stabilisation globale de la symptomatologie. L'atteinte respiratoire restant sévère (ventilation 5 heures/nuit, pas nécessaire pendant la sieste), 6 cures d'ENDOXAN ${ }^{\circledR}$ ont été proposées en 2009, ce d'autant que les EMG de contrôle retrouvaient un tracé appauvri sur les quadriceps et jambiers antérieurs, le tout faisant discuter d'une atteinte neurogène témoin d'une possible aggravation.

Des complications à type d'infections urinaires les ont fait suspendre après six cures.

Un nouvel EMG en 2011 retrouvait un bloc de conduction sur le nerf médian droit à l'avant-bras et les blocs antérieurement décrits à l'avant-bras sur les nerfs ulnaires. Les CPK étaient alors à 295 unités/1 (Tableau 1).

Un relais par immunosuppresseurs per os de type CELLCEPT $^{\circledast}$ avait été instauré puis arrêté en 2015 $\mathrm{du}$ fait de surinfections pulmonaires fréquentes. 
En 2016, les immunoglobulines polyvalentes étaient reprises et un nouveau bilan étiologique était décidé. Dans le cadre de ce bilan, le dosage de l'activité maltase acide sur papier-buvard était diminué à 2,3 pmol/tache/heure (normes 6,0-25,0) avec un rapport maltase/hexosaminidase x 1000 à 0,9 (norme $>3,0$ ). La confirmation sur lymphocytes retrouvait activité une activité maltase acide nettement diminuée à 1,1 (normes 4,9-13,3) et une maltase inhibée par les anticorps à 0 (norme 2.6-10). L'étude du gène GAA en biologie moléculaire retrouvait deux mutations pathogènes : c. 3213T>G et c. $1437+2 \mathrm{~T}>\mathrm{C}$ confirmant de manière formelle l'existence d'une maladie de Pompe à début tardif (LOPD pour late-onset Pompe Disease) chez ce patient. Un traitement par enzymothérapie substitutive par Myozyme $^{\circledR}$ a été débuté en juin 2017. Un dépistage systématiques des autres complications a été réalisé [cardiaque, ophtalmologique, cérébrale (anévrysme)...]. Le traitement par immunoglobulines intraveineuses a été repris un an après devant l'aggravation des paramètres EMG (Tableau 1).

\section{Commentaire}

La maladie de Pompe à début tardif (LOPD) est la forme la plus fréquente débutant dans la troisième décennie. Le tableau clinique typique associe une faiblesse musculaire des membres à prédominance proximale, une atteinte axiale et une insuffisance respiratoire en rapport avec une atteinte diaphragmatique. L'atteinte respiratoire nécessite le plus souvent le recours à une ventilation non invasive nocturne. Il n'y a habituellement pas d'atteinte cardiaque.

Dans la littérature, on trouve quelques cas de neuropathie associées à la maladie de Pompe avec ou sans corrélations anatomopathologiques de dépôts de glycogène. Un patient d'une grande famille costaricaine [1] présentait des CPK à 112, des activités spontanées anormales diffuses (étages facial, cervico-thoraco-lombaires) et une polyneuropathie sensitivo-motrice à prédominance démyélinisante mais possiblement associée à un diabète et à une neuropathie de réanimation. De manière plus intéressante par rapport à notre observation, il a été retrouvé sur des études autopsiques de patients Pompe des dépôts de glycogène dans les cellules de Schwann au niveau de la moelle épinière, des nerfs sensitifs périphériques et du nerf phrénique [2] mais sans description clinique de telles atteintes chez les patients de leur vivant. Seul un troisième article rapporte la survenue d'une neuropathie motrice aiguë chez un enfant combinée à la mise en évidence d'une atteinte motrice à l'autopsie : la ponction lombaire était normale, l'EMG retrouvait une diminution des amplitudes motrices avec abolition des ondes $\mathrm{F}$ (latences et vitesses normales), sans atteinte sensitive. À l'aiguille, on retrouvait des activités spontanées anormales dans les territoires déficitaires évoquant une neuronopathie motrice. La patiente avait également une atteinte respiratoire sévère nécessitant une ventilation mécanique [3].

Il n'a pas été retrouvé chez notre patient d'épaississements nerveux en regard des blocs de conduction, qui auraient pu faire évoquer des dépôts glycogéniques. Malgré la normalité de la ponction lombaire, l'hypothèse auto-immune reste pour nous l'hypothèse la plus vraisemblable. Nous aurions donc affaire à une double pathologie: une authentique maladie de Pompe à début tardif et une neuropathie autoimmune surajoutée.

Quoi qu'il en soit, l'atteinte respiratoire sévère dans une neuropathie auto-immune, d'autant plus motrice pure, doit rester un point d'appel important et faire pratiquer le dosage de la maltase acide. Il faudrait aussi pratiquer des EMG étagés chez les patients Pompe traités qui s'aggravent à la recherche d'une neuropathie surajoutée.

Initialement, on retrouvait des vitesses de conduction sensitives et amplitudes des réponses normales aux quatre membres. Les vitesses de conduction motrice, latences distales motrices et amplitudes étaient normales dans le territoire des nerfs médians et sciatiques poplités externes. On notait un ralentissement modéré sur les nerfs ulnaires avec une réduction d'amplitude à ce niveau par stimulation proximale évoquant une dispersion à l'avant-bras (anastomose de Martin-Grüber exclue). Aucune anomalie n'était notée en détection à l'aiguille avec des tracés interférentiels sans activité spontanée anormale de repos.

Il n'y avait pas de bloc neuromusculaire. Les derniers EMG en 2017 confirmaient une neuropathie démyélinisante inhomogène, avec des sensitives normales (amplitudes et vitesses), aggravée sur les amplitudes motrices sur les nerfs tibiaux et fibulaires un an après malgré le traitement de substitution par Myozyme ${ }^{\circledR}$.

Pompe disease and motor neuropathy: coincidental association?

\section{LIENS D'INTÉR $\hat{E}$ T}

Les auteurs déclarent n'avoir aucun lien d'intérêt concernant les données publiées dans cet article.

\section{RÉFÉRENCES}

1. Torrealba-Acosta G, Rodríguez-Roblero MC, BogantesLedezma S, et al. First clinical and genetic description of a family diagnosed with late-onset Pompe disease from Costa Rica. Neuromuscul Disord 2017 ; 27 : 951-55.

2. Hobson-Webb LD, Proia AD, Thurberg BL, et al. Autopsy findings in late-onset Pompe disease: a case report and systematic review of the literature. Mol Genet Metab 2012 ; 106 : 462-9. 3. Burrow TA, Bailey LA, Kinnett DG, Hopkin RJ. Acute progression of neuromuscular findings in infantile Pompe disease. Pediatr Neurol 2010 ; 42 : 455-8. 


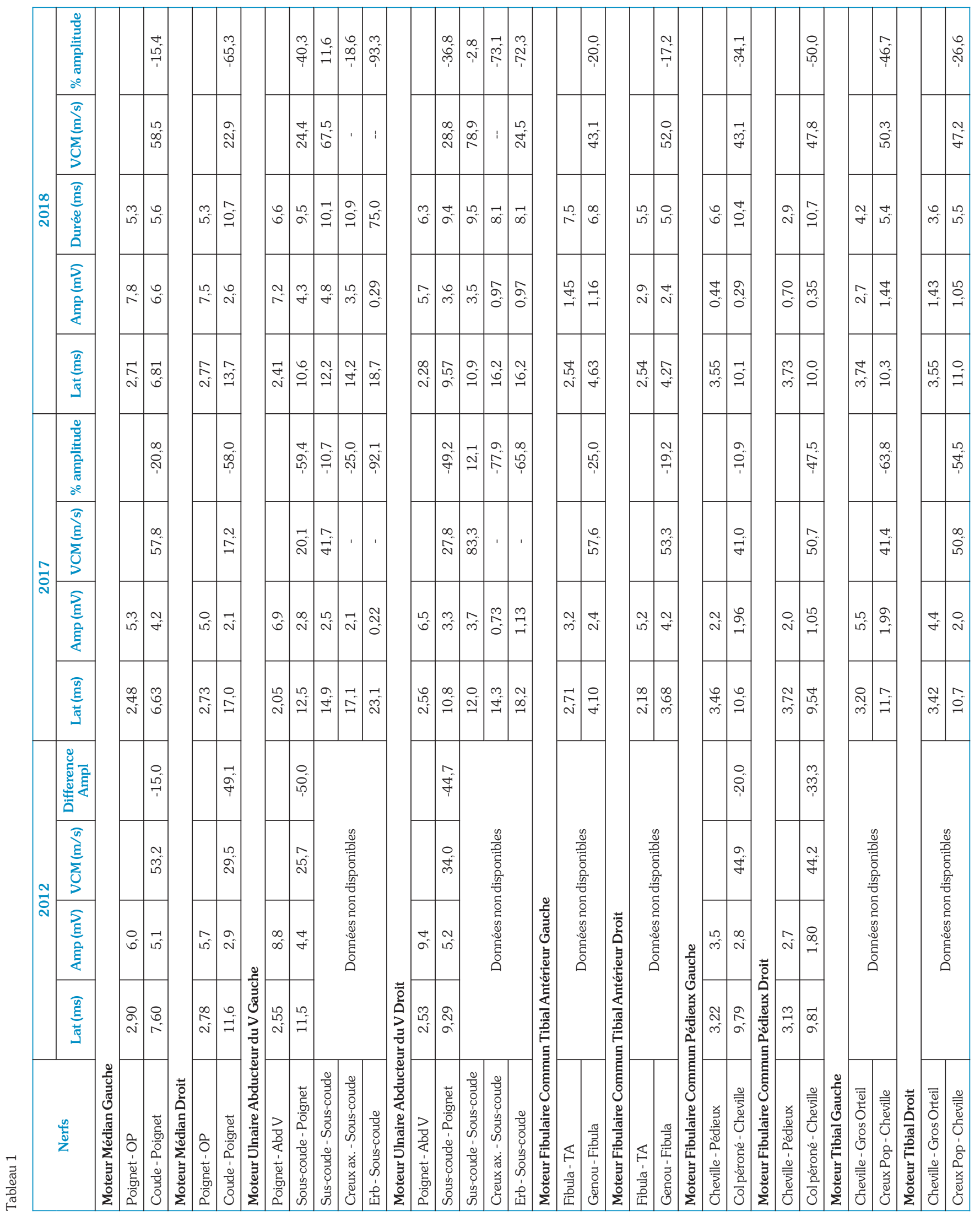

